\title{
Characterization of Microring Filters for Differential Group Delay Applications
}

\author{
D. Doménech, P. Chamorro-Posada, F. J. Fraile-Pelaez, M. J. Erro, S. Tainta, M. A. Muriel, R. Baños, J. Bolten, \\ and H. Kleinjans
}

\begin{abstract}
Index Terms-Integrated optics, longitudinal offset couplers, optical filters, microring resonators, microwave photonics.

Abstract-The longitudinal offset technique permits to improve the accuracy of the coupling coefficients of integrated directional couplers and provides designs that can be easily implemented with current fabrication tolerances. In this work, we address the additional degree of freedom offered by this technology in order to tailor the differential group delay in coupled-resonator optical filters. We present the characterization of several devices exploiting this feature and we discuss their potential applications.
\end{abstract}

\section{INTRODUCTION}

Over the last few years, many photonic signal processing concepts have made their way from the laboratories to the real world, notably thanks to the recent advances in the field of microwave photonics [1]. In this context, microring devices have reached a prominent status as a key technology for the implementation of both linear [2], [3], [4], [5], [6], [7] and nonlinear [8], [9] functionalities. In particular, due to their versatility, coupled resonator optical waveguides (CROW) [10] find many applications in the control of the group delay [11], [12].

At the top-level design, the coupling constants are fundamental parameters. Whereas ring or disk geometries typically permit to obtain larger Q-factors, racetrack microresonators offer superior versatility in the coupling to access waveguides [13], [14], [15] and are often the preferred option. For this configuration, coupling coeficients are determined by the transversal separation between the coupler waveguides in the standard fabrication process of the structures. To meet the

This work has been founded by MINECO (Spanish Government), project numbers TEC2010-21303-C01, 02, 03 and 04, and JCyL Project No. VA089U16. F. J. F.-P. has also been partly supported by the ERDF and by the Galician Regional Government under project GRC2015/018 and under agreement for funding AtlantTIC.

D. Doménech is with VLC Photonics S. L., Camino de Vera s/n, 46022 Valencia, Spain.

P. Chamorro-Posada is with the Departamento de Teoría de la Señal y Comunicaciones e Ingeniería Telemática, Universidad de Valladolid, ETSI Telecomunicación, Paseo Belén 15, E-47011 Valladolid, Spain.

F. J. Fraile-Pelaez is with Dept. Teoría de la Señal y Comunicaciones, Universidad de Vigo, ETSI Telecomunicación, Campus Universitario, E-36310 Vigo (Pontevedra), Spain.

M. J. Erro and S. Tainta are with Department of Electrical and Electronic Engineering, Universidad Pública de Navarra, 31006 Pamplona, Spain.

M. A. Muriel is with Departament of Photonic Technology, Universidad Politécnica de Madrid, 28040 Madrid, Spain.

R. Baños is with Universitat Politecnica de Valencia, iTEAM, Camino de Vera s/n, 460222 Valencia, Spain.

J. Bolten and H. Kleinjans are with AMO GmbH, Otto-Blumenthal-Straße 25, 52074 Aachen, Germany. typically required accuracy levels, such separation must be set with a precision of around a few nanometers, bordering technological limits. To overcome this difficulty, an alternative approach was proposed in [16], [17], which consists in varying the coupling length rather than the inter-guide separation, by changing the longitudinal offset between the two parallel coupled straight waveguides of fixed length. Photolitographic production is then feasible as the resolution requirements are strongly relaxed.

As was highlighted in [18], there exists an asymmetry in the phase transmission characteristics of longitudinal offset structures that opens up new possibilities for the design of photonic devices based on the control of the associated differential group delay. Several structures were proposed in [18] and their experimental demonstration is presented here.

\section{TRANSMisSiOn PROPERTIES}

The two sets of input/output ports available in a longitudinal offset CROW are respectively marked as $A$ and $B$ in Figure 1 for a three-ring system. The associated transmission paths for the fast and slow port sets are identified with dashed and solid arrows, respectively. The corresponding transfer functions are [18]

$$
\begin{array}{r}
H_{A}(\omega)=\exp \left(j \tau_{\text {off }} \omega\right) H(\omega) \\
H_{B}(\omega)=\exp \left(-j \tau_{\text {off }} \omega\right) H(\omega) .
\end{array}
$$

$H(\omega)$ is a common transmission term that can be evaluated [18] as $1 / m_{11}$ in the expression

$$
\left(\begin{array}{ll}
m_{11} & m_{12} \\
m_{21} & m_{22}
\end{array}\right)=\prod_{l=0}^{N} \frac{1}{\left(j t_{l}\right)}\left(\begin{array}{cc}
-\exp (j \beta L) & r_{l} \\
-r_{l} & \exp (-j \beta L)
\end{array}\right),
$$

where $L$ is one half of the total ring length, which is assumed to be constant in the structure, $\beta$ is the propagation constant, and $r_{l}$ and $t_{l}$ are, respectively, the bar and cross coupling coefficients for the optical field at coupler $l$.

The differential group delay parameter is defined as

$$
\tau_{\text {off }}=n_{g} / c \sum_{l=0}^{N} L_{\text {off }, l},
$$

where $n_{g}$ is the group index in the optical waveguides, and $L_{\text {off }, l}$ is the longitudinal offset for coupler $l . \tau_{\text {off }}$ can be identified in Figure 1 as the net propagation path difference due to the longitudinal offsets in the structure with no resonance enhancement. Therefore, this differential delay is limited by the ring size and the number of resonantors. A given coupling 


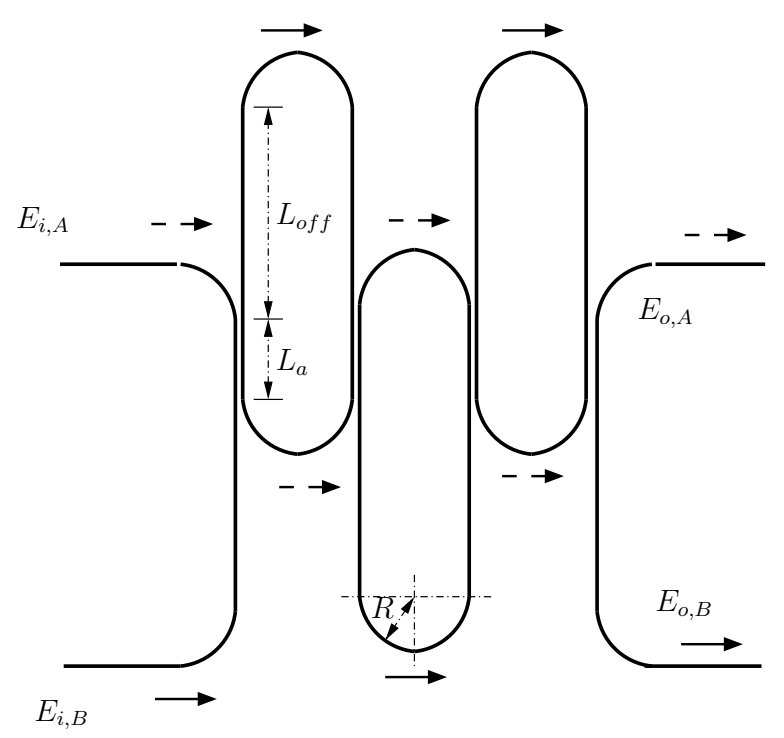

Fig. 1. Geometry of a three-ring longitudinal offset CROW implementing two transfer functions with distinct group delays.

coefficient can be typically obtained at different offset lengths, permitting to adjust the total differential group delay.

\section{FABRICATION AND CHARACTERIZATION}

As described below, the foundry has an optimized process for the lithography and for the etching which ensures high quality in the sidewall roughness and guaranteed critical dimensions. The critical dimensions check ensures the control of the gaps during the fabrication process.

Electron Beam Lithography (EBL) and dry etching were used to fabricate the device by a process similar to that described in [19]. In this case, the silicon-on-insulator (SOI) substrate consists of a $3 \mu \mathrm{m}$ thick buried oxide layer and a 220 $\mathrm{nm}$ thick Si layer. In the first step of the fabrication, the device features are defined by employing hydrogen silsesquioxane negative tone resist and a high contrast development process [20]. Using an HBr-based ICP-RIE process, they are fully etched to the buried oxide [21]. The second step involves the careful aligning of a positive tone ZEP resist mask to the previously defined features. The mask is used to define the shallow etched parts of the devices, and C4F8/SF6-based dry etching is employed.

Aiming to minimize the scattering losses of the devices, multi-pass exposure is used in both steps in order to reduce the sidewall roughness further. A very accurate proximity effect correction is also needed to guarantee the accuracy in the critical dimensions of the devices, in combination with a suitable exposure dose [22].

The wire waveguides are $450 \mathrm{~nm}$ wide and $220 \mathrm{~nm}$ thick to ensure TE monomode propagation. The measured linear propagation loss in such photonic wires is $3 \pm 0.5 \mathrm{~dB} / \mathrm{cm}$.

The resolution of the e-Beam lithography employed is below $10 \mathrm{~nm}$, thus leading to a very accurate control of the coupling gaps between rings. As illustrated in Figure 2, the coupling constant uncertainty can be controlled below $10 \%$. The longitudinal offset technique has been applied in order

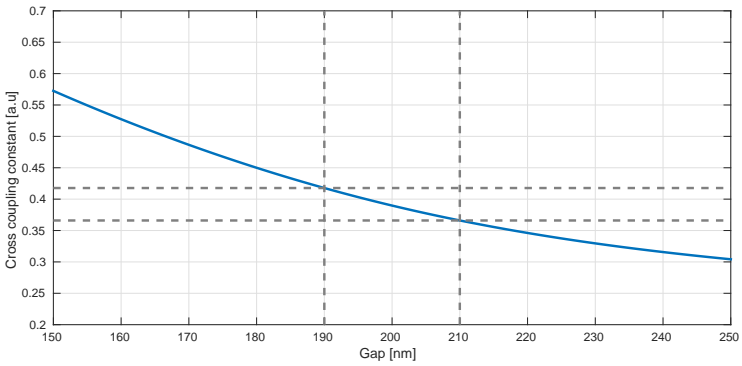

Fig. 2. Tolerance analysis of the coupling constant for a deviation in the gap/waveguide width of the parallel waveguide couplers implemented in the CROW structures. A reduction of $10 \mathrm{~nm}$ in the gap (and the subsequent waveguides width increase) implies a change in the coupling constant of 0.025 , which corresponds to a $6.25 \%$ relative change.

to guarantee the uniformity between cavities since the gap is kept constant for all the rings. The SEM images of the couplers show that the width of the waveguides in the coupler range from $437 \mathrm{~nm}$ to $455 \mathrm{~nm}$, while the measured gaps are in the range from $196 \mathrm{~nm}$ to $214 \mathrm{~nm}$. All these quantities differ from device to device and from die to die.

All input/output waveguides in the chip have been provided with grating couplers, which only support TE polarization [23]. The measurements have been normalized to the response of a straight waveguide in order to remove the spectral response of the grating coupler. For three-port structures (Section 3.1), the insertion losses are $1 \mathrm{~dB} \pm 0.5 \mathrm{~dB}$, while for the twoports device, they are in the range $5 \mathrm{~dB} \pm 2 \mathrm{~dB}$. These values depend greatly on the normalization waveguide employed to remove the filtering effect of the grating coupler.

\section{A. Three-port structures}

We first consider the structure obtained when two ports in the reference structure of Figure 1 are combined using a Ybranch coupler. The resulting geometry is shown in Figure 3, where we have employed an MMI coupler.

This set-up permits to obtain two identically filtered replicae of the input with a relative delay

$$
\Delta \tau=2 \tau_{\text {off }}=2 \frac{n_{g}}{c} \sum_{l=0}^{N} L_{\text {off }, l}
$$

that, in the case of equal $L_{\mathrm{off}, l}=L_{\mathrm{off}}$, reads

$$
\Delta \tau=2(N+1) \frac{L_{\mathrm{off}} n_{g}}{c} .
$$

We have fabricated and characterized three structures with 3,5 and 10 racetrack micro-rings, respectively. In all cases, the bend radius of the racetracks $R=5 \mu \mathrm{m}$, the coupling gaps are $s=0.2 \mu \mathrm{m}, L_{\text {off }}=82.339 \mu \mathrm{m}$ and the total cavity length is $L_{\text {cav }}=2 L=231.42 \mu \mathrm{m}$ with an associated free spectral range of FSR $=c /\left(L_{\text {cav }}\right) \simeq 285 \mathrm{GHz}$. The coupling constant at a reference wavelength $\lambda=1.55 \mu \mathrm{m}$ is $K=|t|^{2}=0.64$.

Using (5), the differential delay between outputs are $9.99 \mathrm{ps,}$ $14.98 \mathrm{ps}$ and $27.47 \mathrm{ps}$ for 3,5 and 10 rings structures, respectively. Figure 4 (a) compares the measured signals as transmitted in the fast and slow paths at four different values of the 


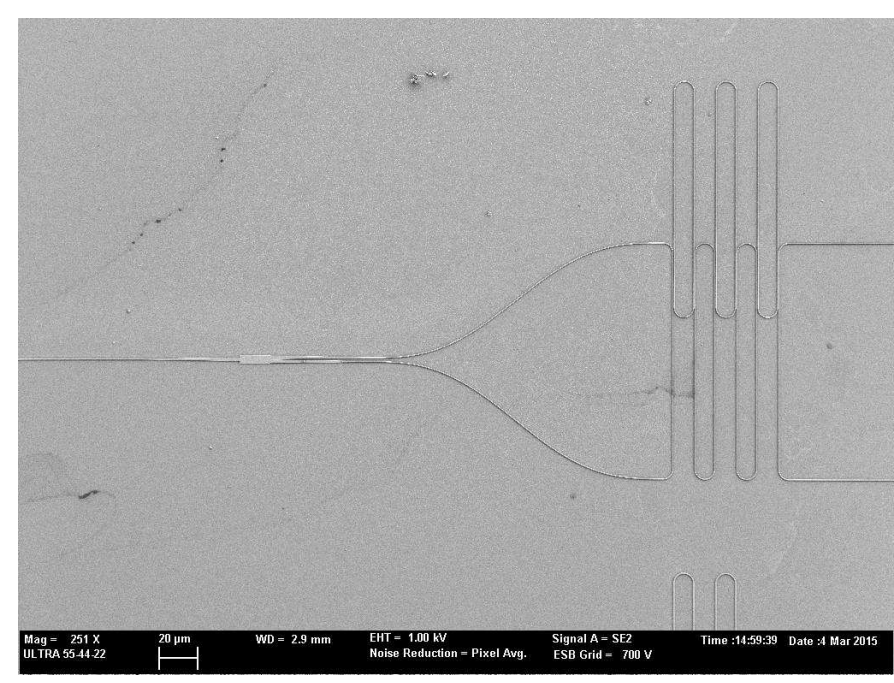

Fig. 3. SEM image of the fabricated structure producing two output replicae with a controlled relative group delay with five racetracks.

carrier frequency in the filter pass-bands. Ten measurements have been performed per wavelength to estimate the average and the standard deviation of the group delay. Figure 4 (b), shows the excellent agreement between experiment and theory.

This structure can be cascaded and could find application, for instance, in optical beam-forming networks [24] as discussed in [18]. Reversing the input and output ports, the structures can also be employed for simultaneously implementing a precise filtering operation through $H(\omega)$ and obtaining a timedomain multiplexed output signal from two input signals.

\section{B. Two-port structures}

New design possibilities are found when both the input and output ports are connected using Y couplers. If $\Delta \tau$ is greater than the pulse duration the addition of a phase control in one of the output paths, for instance, can be used [18] for the production and processing of time-bin encoded states for quantum information applications [25], [26], [27]. In this case we have a compact integrated set-up incorporating the precise waveform shaping provided by the filtering in the CROW.

The combination of ports, both at the input and output of the device in a Mach-Zehnder configuration, can also be used to double the FSR of a given structure without significantly affecting the bandwidth and introducing additional transmission nulls between resonances. If we assume the same input in Eqs. (1) and combine them into a single output, we obtain the transfer function

$$
H_{\mathrm{MZ}}(\omega)=\cos \left(\tau_{\mathrm{off}} \omega\right) H(\omega) .
$$

To double the FSR, the position of a spectral null every other transmission band of the CROW filter requires the fulfillment of the condition $\tau=4 \tau_{\text {off }}$ or, for constant $L_{\text {off }, l}=L_{\text {off }}$,

$$
L_{\text {off }}=\frac{L}{2(N+1)} .
$$

(a)

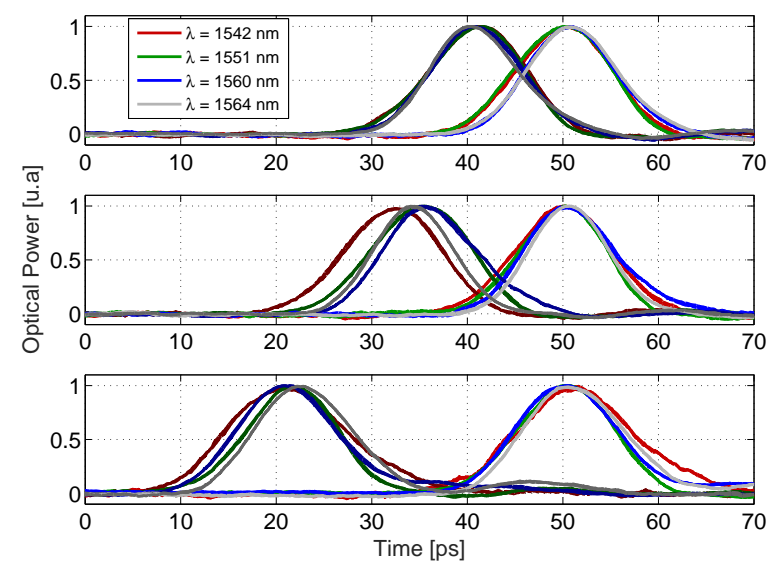

(b)

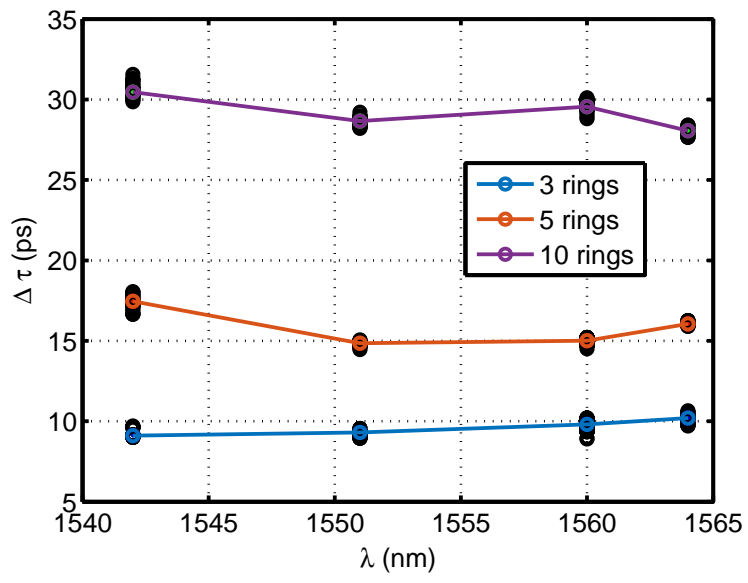

Fig. 4. (a) Measured output pulse intensities at four different wavelengths for three (top) five (middle) and ten (bottom) rings. (b) Average group delay and its standard deviation for each structure.

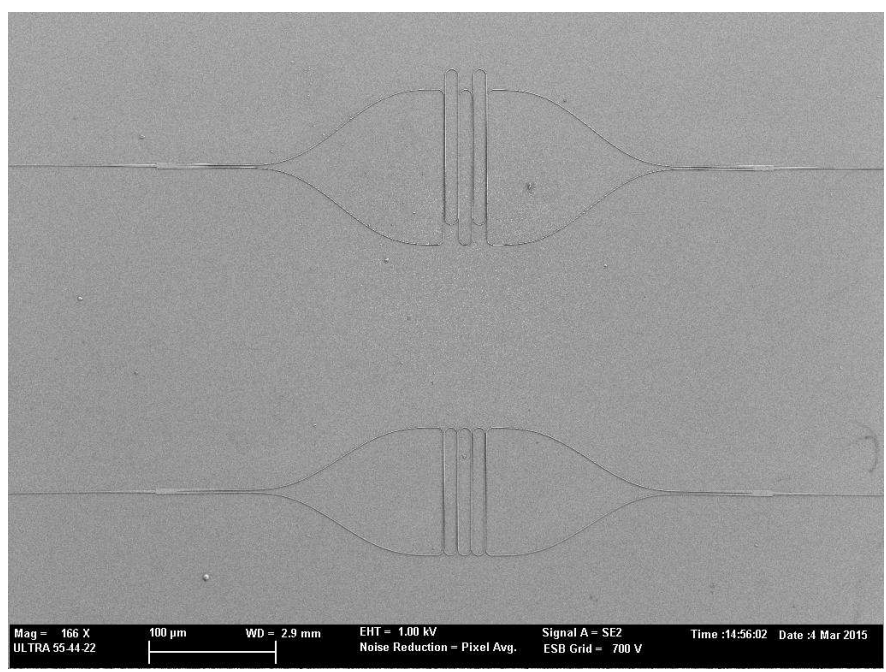

Fig. 5. SEM image of the fabricated and characterized 3-ring MZ configuration devices with (top) and without (bottom) longitudinal offset couplers.

The one-half resonator length is $L=L_{a}+L_{\text {off }}+\pi R$, where $L_{s}=L_{a}+L_{\text {off }}$ is the total length of the straight waveguide 


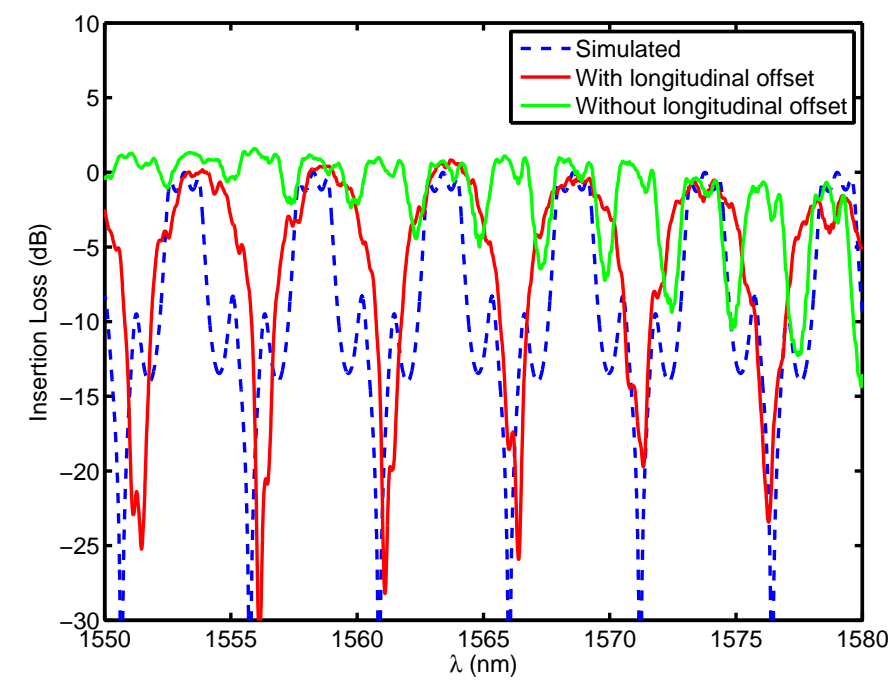

Fig. 6. Measured transmission response of a three-ring Mach-Zehnder device configuration with and without longitudinal offset couplers and the corresponding calculated response with longitudinal offsets. This corresponds to the case with $s=0.3 \mu \mathrm{m}$ and other parameters as described in the text.

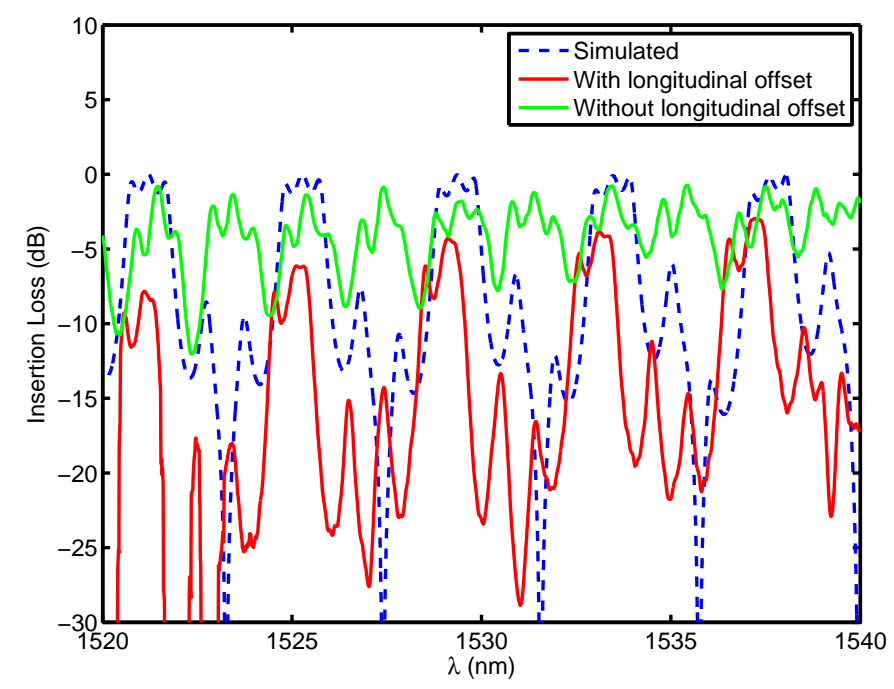

Fig. 7. Same as Figure 5 for the robust design case with $s=0.35 \mu \mathrm{m}$ and other parameters as described in the text.

sections. Therefore, the above condition reads

$$
L_{\mathrm{off}}=\frac{L_{a}+\pi R}{2 N+1} .
$$

The measured transmission responses of two MZ configurations with and without longitudinal offset differential group delay, as displayed in Figure 5, are compared in Figure 6 along with the transfer function computed using coupled mode theory. In this case $s=0.3 \mu \mathrm{m}, L_{s}=88.233 \mu \mathrm{m}$ and $L_{\text {off }}=$ $12.993 \mu \mathrm{m}$. The cavity $Q$ factor for the device with differential group delay is found to be $Q \simeq 650$. The measurements clearly show the expected FSR doubling in the structure. When comparing the results for the MZ configurations with and without differential group delay, it is important to bear in mind that the introduction of the longitudinal offset modifies the coupling constant in the structure.
If the design based on the condition (8) is too sensitive to fabrication tolerances, an alternative, more robust, design can be obtained for a limited bandwidth [18]. If $\tau=p \tau_{\text {off }}$, with $p$ a rational number close to 4 , the MZ and CROW resonance conditions will coincide in a particular band of the CROW periodic response, and an approximate cancellation will happen in a certain bandwidth that will depend on the value of $p$. In this case, the value of a constant offset for a given coupler length is given by the expression

$$
L_{\text {off }}=\frac{2 L_{a}+2 \pi R}{(N+1) p-2} .
$$

Figure 7 shows the experimental and simulated results for $s=0.35 \mu \mathrm{m}, L_{s}=109.558 \mu \mathrm{m}, L_{\text {off }}=15.408 \mu \mathrm{m}$, and $p=1000 / 256=4.065$. Here the cavity $Q$ factor is found to be $Q \simeq 520$. In this case, the FSR doubling has been achieved for a $20 \mathrm{~nm}$ bandwidth.

\section{CONCLUSION}

We have fabricated and characterized various structures exploiting the inherent differential group delay available in CROW chains realized by means of the longitudinal offset technique. Three port networks, obtained by combining either the input or output ports of the fast and slow channels, and two port networks, with port combination taking place both at the input and the otuput, have been demonstrated. Three port networks could find application, for instance, for combining filtering and time-domain multiplexation operations in integrated optical circuits applications or as building blocks for complex group delay networks that could be used in optical beam-forming antennas. The two port devices could be used for quantum information time-bin encoding applications for differential delays of the order of the pulse duration. We have demonstrated a more general set-up for FSR doubling in quasi-periodic filters. Simultaneous filtering and relative delaying operations can also be performed combining cascaded bandpass filters and waveguide delay pairs. In large integrated circuits with extensive use of this type of operations, our proposal permits to obtain more compact designs and a relevant reduction of the number of elements. In Quantum Information applications [27], the reduction of the complexity and the ellimination of off-circuit filters has a direct impact on the photon loss, which is critical for the device operation, and avoids asymmetries that can also affect system performance.

\section{REFERENCES}

[1] J. Capmany, J. Mora, I. Gasulla, J. Sancho, J. Lloret, and S. Sales, "Microwave photonic signal processing," J. Lightwave Technol., vol. 4, no. 31, pp. 571-586, Feb. 2013.

[2] G. Lenz and C.K. Masden, "General optical all-pass filter structures for dispersion control in WDM systems," J. Lightwave Technol., vol. 17, no. 7, pp. 1248-1254, Jul. 1999.

[3] P. Chamorro-Posada, F.J. Fraile-Pelaez, and F. J. Diaz-Otero, "Microring chains with high-order resonances," J. Lightwave Technol., vol. 29, no. 10, pp. 1514-1521, May. 2011.

[4] C. K. Madsen, G. Lenz, A. J. Bruce, M. A. Cappuzzo, L. T. Gomez, and R. E. Scotti, "Integrated all-pass filters for tunable dispersion and dispersion slope compensation," IEEE Photon. Technol. Lett., vol. 11, no. 12, pp. 1623-1625, Dec. 1999.

[5] C. Y. Chao, W. Fung, and L. J. Guo, "Polymer microring resonators for biochemical sensing applications," IEEE J. Sel. Top. Quantum Electron., vol. 12, no. 1, pp. 134-142, Jan./Feb. 2006. 
[6] R. W. Boyd, D. J. Gauthier, and A. L. Gaeta, "Applications of Slow Light in Telecommunications," Opt. Photonics News, vol. 17, no. 4, pp. 18-23, Apr. 2006.

[7] P. Chamorro-Posada and F. J. Fraile-Pelaez, "Fast and slow light in zigzag microring resonator chains," Opt. Lett., vol. 34, no. 5, pp. 626-628, Mar. 2009.

[8] R. Gómez-Alcalá, F. J. Fraile-Pelaez, P. Chamorro-Posada, and F. J. Díaz-Otero, "Modeling of racetrack-resonator add-drop filters with arbitrary nonlinear directional couplers," Opt. Lett., vol. 37, no. 11, pp. 2097-29099, Jun. 2012.

[9] V. Grigoriev and F. Biancalana, "Resonant self-pulsations in coupled nonlinear microcavities," Phys. Rev. A, vol. 80, no. 4, p. 043816, Apr 2011.

[10] A. Yariv, Y. Xu, R. K. Lee and A. Scherer, "Coupled-resonator optical waveguide: a proposal and analysis," Opt. Lett., vol. 24, no. 11, pp. 711-713, Jun. 1999

[11] J. E. Heebner and R. W. Boyd, "Slow and fast light in resonator-coupled waveguides," J. Mod. Opt., vol. 49, no. 14/15, pp. 2629-2636, Nov./Dec. 2002.

[12] J. K. Poon, L. Zhu, G. A. DeRose, and A. Yariv, "Transmission and group delay of microring coupled-resonator optical waveguides," Opt. Lett., vol. 31, no. 4, pp. 456-458, Feb. 2006.

[13] W. Bogaerts, P. DeHeyn, T. Van Vaerenbergh, K. De Vos, S. K. Selvaraja, T. Claes, P. Dumon, P. Bienstaman, D. Van Thourhout, and R. Baets, "Silicon microring resonators," Laser Photonics Rev., vol. 6, no. 1, pp. 47-73, Dec. 2011.

[14] H. A. Haus, M. A. Popović, M. R. Watts, C. Manolatou, B. E. Little, and S. T . Chu, "Optical resonators and filters," in Optical Microcavities, K. Vahala. Singapore: World Scientific, 2004, pp. 1-37.

[15] P. Chamorro-Posada, "Q-enhanced racetrack microresonators," Opt. Comm., vol. 387, pp. 70-78, Mar. 2017.

[16] J. D. Doménech, P. Muñoz, and J. Capmany, "The longitudinal offset technique for apodization of coupled resonator optical waveguide devices: concept and fabrication tolerance analysis," Opt. Express, vol. 17, no. 23, pp. 21050-21059, Nov. 2009.

[17] J.D. Doménech, P. Muñoz, and J. Capmany, "Transmission and groupdelay characterization of coupled resonator optical waveguides apodized through the longitudinal offset technique," Opt. Lett., vol. 36, no. 2, pp. 136-138, Jan. 2011.

[18] P. Chamorro-Posada and F. J. Fraile-Pelaez, "Group delay control in longitudinal offset coupled resonator optical waveguides," J. Lightwave Technol., vol. 33, no. 9, pp. 1703-1707, Aug. 2014.

[19] B. Gargallo, P. Muñoz, R. Baños, A. L. Giesecke, J. Bolten, T. Wahlbrink, and $\mathrm{H}$. Kleinjans, "Reflective arrayed waveguide gratings based on Sagnac loop reflectors with custom spectral response," Opt. Express, vol. 22, no. 12, pp. 14348-14362, Jun. 2014.

[20] W. Henschel, Y. M. Georgiev, and H. Kurz, "Study of a high contrast process for hydrogen silsesquioxane as a negative tone electron beam resist," J. Vac. Sci. Technol. B, vol. 21, no. 5, pp. 2018-2025, Sept. 2003.

[21] M. C. Lemme, T. Mollenhauer, H. D. G. Gottlob, W. Henschel, J. Efavi, C. Welch, and H. Kurz, "Highly selective $\mathrm{HBr}$ etch process for fabrication of Triple-Gate nano-scale SOI-MOSFETs," Microelec. Eng., vol. 73/74, pp. 346-350, Jun. 2004.

[22] J. Bolten, T. Wahlbrink, N. Koo, H. Kurz, S. Stammberger, U. Hofmann, and N. Ünal, "Improved CD control and line edge roughness in E-beam lithography through combining proximity effect correction with gray scale techniques," Microelec. Eng., vol. 8, pp. 1041-1043, Aug. 2010.

[23] D. Taillaert, F. Van Laere, M. Ayre, W. Bogaerts, D. Van Thourhout, P. Bienstman, and R. Baets, "Grating Couplers for Coupling between Optical Fibers and Nanophotonic Waveguides," Japanese J. of Appl. Phys., vol. 45, no. 1-8A, pp. 6071-6077, Aug. 2006.

[24] L. Zhuang, C. G. H. Roeloffzen, R. G. Heideman, A. Borreman , A Meijerink, and W. van Etten, "Single-chip ring resonator-based $1 \times$ 8 Optical Beam Forming Network in CMOS-Compatible Waveguide Technology," IEEE Photon. Technol. Lett., vol. 19, no. 15, pp. 11301132, Aug. 2007.

[25] J. Brendel, N. Gisin, W. Tittel, and H. Zbinden, "Pulsed Energy-Time Entangled Twin-Photon Source for Quantum Communication," Phys. Rev. Lett., vol. 83, no. 12, pp. 2594-2597, March 1999.

[26] W. Tittel, J. Brendel, H. Zbinden, and N. Gisin, "Quantum Cryptography Using Entangled Photons in Energy-Time Bell States," Phys. Rev. Lett. vol. 84, no. 20, pp. 4737-4740, May 2000.

[27] C. Xiong, et al "Compact and reconfigurable silicon nitride time-bin entanglement circuit," Optica, vol. 2, no. 2, pp. 724-727, Aug. 2015. 